\title{
Towards measuring the off-resonant thermal noise of a pendulum mirror
}

\author{
V Leonhardt ${ }^{1}$, L Ribichini ${ }^{2}$, P Klövekorn ${ }^{2,3}$, B Willke ${ }^{1,2}$, H Lück $^{1,2}$ \\ and K Danzmann ${ }^{1,2}$ \\ ${ }^{1}$ Institut für Atom- und Molekülphysik, Universität Hannover, Callinstrasse 38, D-30167 \\ Hannover, Germany \\ 2 Albert Einstein Institut für Gravitationsphysik, Aussenstelle Hannover, Callinstrasse 38, \\ D-30167 Hannover, Germany
}

Received 26 September 2001, in final form 24 October 2001

Published 14 March 2002

Online at stacks.iop.org/CQG/19/1717

\begin{abstract}
Thermal noise is one of the dominant noise sources in interferometric length measurements and can limit the sensitivity of gravitational wave detectors. Our goal is to analyse the off-resonant thermal noise of a high $Q$ pendulum. Therefore we interferometrically detect the length changes of a $2.3 \mathrm{~cm}$ long optical resonator, which for good seismic isolation consists of two multiple stage pendulums. We are able to lock the length of this optical resonator to a frequency-stabilized laser beam and as a result get the spectral density of the differential mirror movement.
\end{abstract}

PACS numbers: $0480 \mathrm{~N}, 9555 \mathrm{Y}$

\section{Introduction}

In the large gravitational wave detectors such as TAMA [1], VIRGO [2], LIGO [3] and GEO [4], thermal noise is one of the dominant limits to the achievable sensitivity. Hence it is important to have a better understanding of the thermal noise.

There are some thermal noise measurements far off the resonance of a mechanical oscillator [5,6], but the thermal noise of the pendulum mode of a suspended mirror is yet to be measured. This is a complex issue because a pendulum usually has very low internal friction. The reason for this is that most of the energy of the pendulum's horizontal motion is losslessly stored in the gravitational potential, which leads to very high $Q$-values for mechanical pendulums and to low thermal noise outside the pendulum resonance.

The power spectral density of the thermal noise can be estimated by using the fluctuationdissipation theorem [7]. Its slope depends on how the oscillator loses energy. In the case of a damping force proportional to the velocity, the mechanical oscillator can be described by

$$
m \ddot{x}+\gamma \dot{x}-F_{\text {Spring }}=F_{\text {th }} .
$$

${ }^{3}$ Present address: Fiberbyte, 227 Gouger St, Adelaide, SA 5000, Australia. 


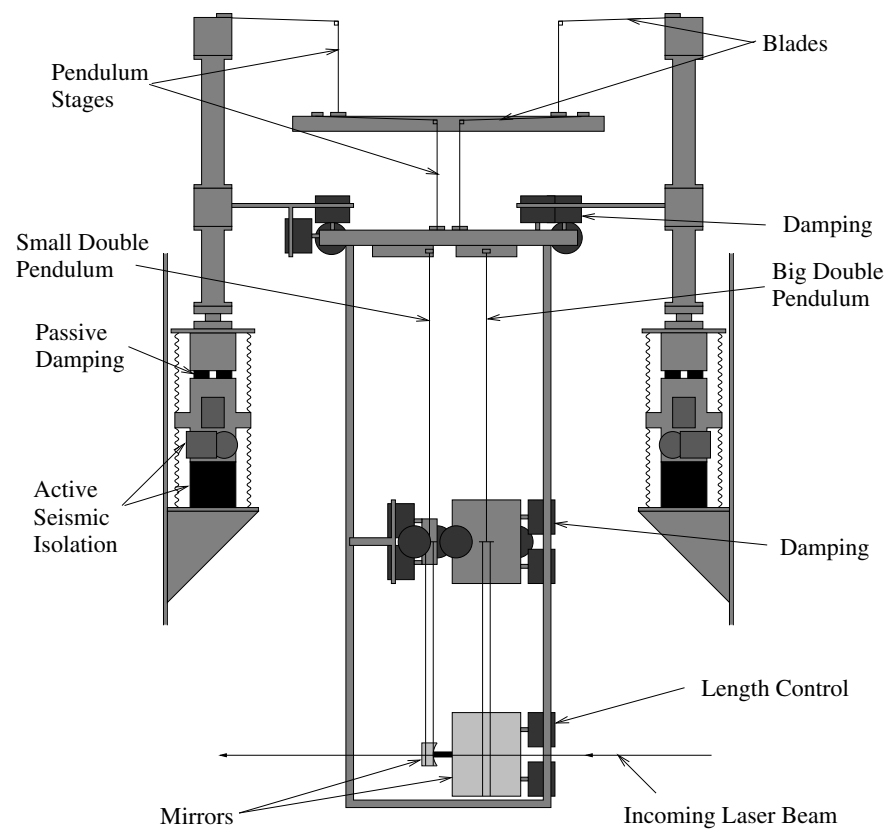

Figure 1. Seismic isolation in our experiment. We have a frame suspended as a double pendulum with blades at both stages. Two mirrors are suspended as double pendulums from this frame. The resonance frequencies of the pendulums are damped by coil-magnet actuators.

$F_{\text {Spring }}=-k x$ is the restoring force and $F_{\text {th }}^{2}(\omega)=4 k_{b} T \gamma$ is the thermal excitation, which has a white spectral density. The resulting mirror movement can be calculated as [8]

$$
x^{2}(\omega)=\frac{4 k_{b} T \gamma}{\left(k-m \omega^{2}\right)^{2}+f^{2} \omega^{2}} .
$$

Internal damping in the material can be described by a restoring force with a complex spring constant where the imaginary part represents the loss of the system

$$
F_{\text {Spring }}=-k[1+\mathrm{i} \phi(\omega)] x .
$$

$\phi(\omega)$ is called the loss function, and its frequency dependence defines the slope of the thermal noise outside the resonance. The resulting spectral density would be

$$
x^{2}(\omega)=\frac{4 k_{b} T k \phi(\omega)}{\omega\left[\left(k-m \omega^{2}\right)^{2}+k^{2} \phi^{2}(\omega)\right]} .
$$

A loss function proportional to $\omega$ would be equivalent to the case of velocity-dependent damping, however $\phi(\omega)$ is believed to have a different frequency dependence [9, 10]. To distinguish which model for $\phi(\omega)$ is closest to reality, it is necessary to measure the slope of the thermal noise outside the resonance.

\section{Experimental set-up}

To achieve a good sensitivity at the frequencies of interest, the seismic noise has to be minimized in a frequency range from 30 to $100 \mathrm{~Hz}$. For this purpose we use an active isolation system which measures seismic motion at frequencies around $2 \mathrm{~Hz}$ and feeds the information 
back to a piezoelectric actuator. After one rubber stage we have a double pendulum with an $18 \mathrm{~kg}$ middle mass. A frame structure of the same weight is suspended from it (see figure 1). Both stages have a length of $24 \mathrm{~cm}$ each. The first consists of four wires with a diameter of $0.8 \mathrm{~mm}$ each while the second stage has two of these wires. For vertical seismic isolation both stages are suspended from blades with resonance frequencies of around $2 \mathrm{~Hz}$.

To avoid the excitation of the double pendulum resonances we measure the movements of the frame using shadow sensors in six degrees of freedom and feed the information back to coil-magnet actuators. The identical masses of the two pendulum stages ensure a good coupling of the eigenmodes of the two stages. Therefore we are able to damp the resonances of the whole double pendulum by only acting on one stage. Feedback is applied in a frequency range from 0.5 to $3 \mathrm{~Hz}$.

Inside the frame we have two double pendulums. These pendulums are damped at their upper masses by four coil-magnet actuators each. The first and second pendulum stages have a length of 50 and $30 \mathrm{~cm}$, respectively. The smaller one of these double pendulums consists of a $50 \mathrm{~g}$ mass, which is suspended by two wires with a diameter of $0.1 \mathrm{~mm}$ and a second stage, which is made of four wires with $0.1 \mathrm{~mm}$ diameter. The mass of the second of these stages is $42 \mathrm{~g}$ with an $8 \mathrm{~g}$ mirror attached to it. The smaller the second mass, the bigger its movement due to thermal noise. The radius of curvature of this mirror is $1 \mathrm{~m}$. The second double pendulum is made of two $900 \mathrm{~g}$ masses. The upper two wires have a diameter of $0.13 \mathrm{~mm}$, while the lower four have a diameter of $0.1 \mathrm{~mm}$. A flat mirror is attached to the big mass and together with the curved mirror it forms a $2.3 \mathrm{~cm}$ long optical resonator. The transmission of both mirrors is $0.1 \%$.

Three magnets are attached to the bigger one of the mirrors. By sending a current through three additional coils at the frame we are able to apply a force to the big mirror and thus change the length of the optical resonator.

The whole experiment is set up in a vacuum tank to avoid excitation of the pendulums by gas molecules. The pressure inside the tank is in the $10^{-7}$ mbar region.

\section{Optical layout}

The length of the suspended resonator is stabilized to the frequency of the incoming laser beam using a Pound-Drever-Hall locking scheme [11] with a modulation frequency of 7.9 $\mathrm{MHz}$ (see figure 2). To avoid back-reflections from the flat surface of the big mirror into the laser two Faraday isolators are necessary.

As long as we have sufficient gain the feedback suppresses the differential mirror movement. Therefore in the low-frequency region we look at the feedback signal. Above the unity gain frequency the error-point signal is proportional to the mirror movement. In our experiment unity gain of the feedback is around $300 \mathrm{~Hz}$. It would be an advantage to shift this to a higher frequency to have more gain in the frequency region of a few $\mathrm{Hz}$ where the mirrors move the most, but internal mechanical resonances of the mirrors prevent us from doing so.

One major noise source in this set-up is the laser-frequency noise. It appears in our Pound-Drever-Hall readout scheme as a length change of the suspended resonator. This effect can be calculated by

$$
\frac{\Delta f}{f}=\frac{\Delta l}{l}
$$

where $\Delta f$ is the change of the laser frequency $f$ and $\Delta l$ is the corresponding change of the resonator length $l$. Keeping $l$ as short as possible we can reduce this effect, but a stabilization of the laser frequency is still necessary. 


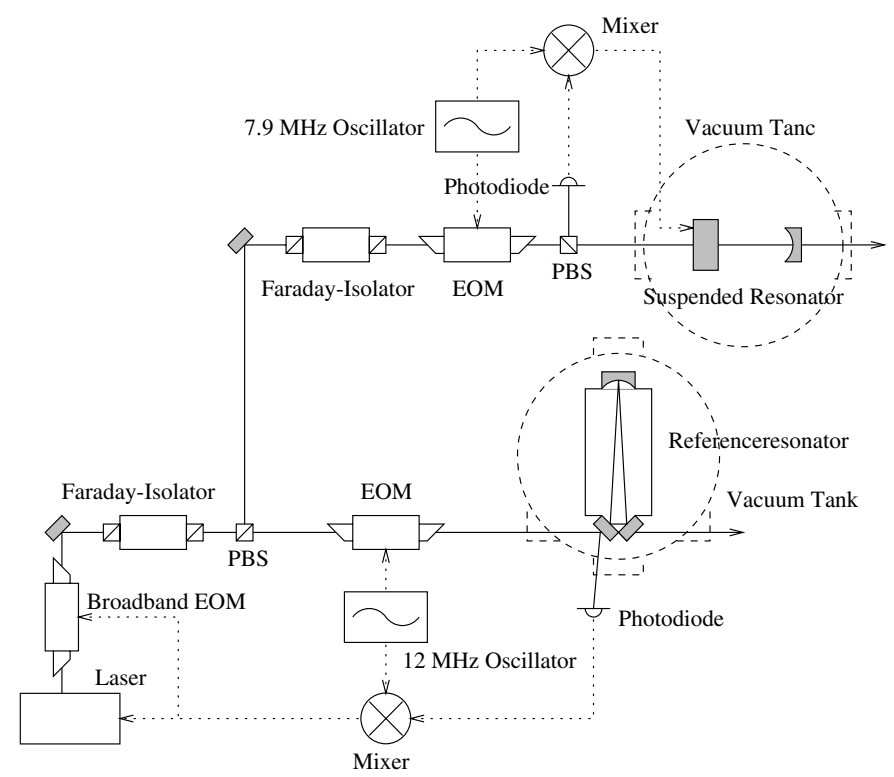

Figure 2. Optical layout. The laser is stabilized to a reference resonator and the length of the suspended resonator is locked to the laser frequency.

To achieve this we use a reference resonator in a separate vacuum tank. This resonator has a finesse of 58000 and an optical path length of $424 \mathrm{~mm}$. For this second Pound-Drever-Hall locking scheme we use a modulation frequency of $12 \mathrm{MHz}$. To actually change the laser frequency we use three actuators. To counter dc frequency drifts we change the temperature of the laser crystal, from low frequencies up to a few $\mathrm{kHz}$ we use a piezoelectric actuator to apply a pressure to the laser crystal, and for frequencies up to $200 \mathrm{kHz}$ we use an electro-optical modulator as a phase shifter [12].

The calibration of the feedback signal is done by applying a sine wave to the piezoelectric actuator on the laser crystal. The stabilization of the laser frequency has to be turned off for this. With the knowledge of the size of the change in the laser frequency due to the applied sine wave, we calculate the corresponding length change of the optical resonator by using equation (5) and thus calibrate the resulting feedback signal.

This method has to be repeated at all frequencies to get a complete frequency-dependent transfer function. The frequency dependence comes from the fact that our coil-magnet actuators are not moving the big mirror directly, they are applying a force instead. That means that the pendulum transfer function of the last pendulum stage appears in our calibration.

\section{Results}

We have achieved a stable lock of our suspended resonator for a time of up to a few hours, which enabled us to measure the spectral density of the differential mirror movement (see figure 3). We can see that the resonance frequencies of the pendulum around $1 \mathrm{~Hz}$ are damped and do not peak much over the $10^{-8} \mathrm{~m} \mathrm{~Hz}^{-1 / 2}$ seismic noise floor of the environment. Above a few $\mathrm{Hz}$, we seem to be limited by the noise of the coil-magnet actuator electronics and the 


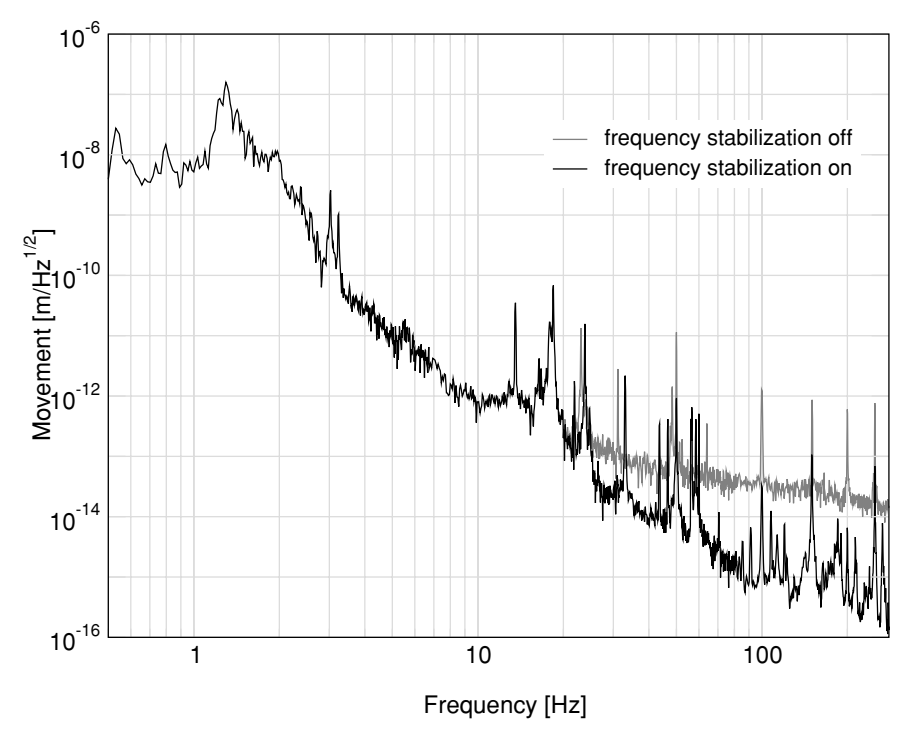

Figure 3. Measured spectral density of the differential mirror movement. Besides some seismically excited resonances, we are limited by electronic noise sources. When the laser frequency stabilization is turned off, the free running laser noise dominates the spectrum at high frequencies.

noise of the feedback for the resonator length. The different peaks above $10 \mathrm{~Hz}$ seem to be seismically excited pendulum resonances in the vertical direction.

To improve the spectrum we are currently working on a new pendulum stage directly above the mirror-to-filter noise from the coil-magnet actuators. Some electronic components also have to be improved. As a next step we will further reduce the mass of the small mirror.

\section{Acknowledgment}

This work was supported by the Deutsche Forschungsgemeinschaft within the Sonderforschungsbereich 407.

\section{References}

[1] Tsubono K (The TAMA collaboration) 1996 Proc. TAMA Workshop on Gravitational Wave Detection (Tokyo: Universal Academy)

[2] Bradaschia C et al 1990 Nucl. Instrum. Methods A 289518

[3] Abramovici A et al 1992 Science 256325

[4] Danzmann K et al 1994 GEO 600_-Proposal for a 600 m laser-interferometric gravitational wave antennax MPQ Garching

[5] González G I and Saulson P R 1995 Phys. Lett. A 20112

[6] Kajima M, Kusumi N, Moriwaki S and Mio K 2000 Phys. Lett. A 264251

[7] Callen H B and Greene R F 1952 Phys. Rev. 86702

[8] Saulson P R 1990 Phys. Rev. D 422437

[9] Saulson P R 1994 Fundamentals of Interferometric Gravitational Wave Detection (Singapore: World Scientific) p 118

[10] Brif C 1999 LIGO Technical Note Ligo T-990041-00-R

[11] Drever R W P, Hall J L, Kowalski F V, Hough J, Ford G M, Munley A J and Ward H 1983 Appl. Phys. B 3197

[12] Nakagawa K, Shelkovnikov A S, Katsuda T and Ohtsu M 1994 Appl. Opt. 336383 